\title{
Entanglement-Assisted Classical Communication Over Quantum Channels for Binary Markov Sources
}

\author{
Mohd Azri Mohd Izhar, Member, IEEE, Zunaira Babar, Member, IEEE, Soon Xin Ng, Senior Member, IEEE, \\ and Lajos Hanzo, Fellow, IEEE
}

\begin{abstract}
Symbol-based iterative decoding is proposed for the transmission of classical Markov source signals over a quantum channel using a three-stage serial concatenation of a convolutional code (CC), a unity-rate code and a two-qubit superdense (SD) protocol. A modified symbol-based maximum a posteriori algorithm is employed for CC decoding to exploit the Markov source statistics during the iterative decoding process. Extrinsic information transfer chart analysis is performed to evaluate the benefit of the extrinsic mutual information gleaned from the $\mathrm{CC}$ decoder for sources with different correlations. We evaluate the bit error rate performance of the proposed coding scheme and compare it to the relevant benchmark schemes, including the turbo coding-based SD scheme. We demonstrate that a nearcapacity performance can be achieved using the proposed scheme and when utilizing sources having a high correlation coefficient of $\rho=0.9$, the proposed coding scheme performs within $0.53 \mathrm{~dB}$ from the entanglement-assisted classical capacity.
\end{abstract}

Index Terms-Entanglement-assisted classical communication, superdense coding, joint source-channel coding, source-assisted channel decoding, iterative decoding.

\section{INTRODUCTION}

Vehicular communications (VCs) enable a variety of exciting applications that can make driving safer, more efficient and more pleasant. However, despite the unique features offered, formidable abuses and attacks are possible that can implicate the benefits of their deployment [1]. For instance, an attacker may intercept, corrupt and manipulate critical messages such as traffic notifications or hazard warnings. Therefore, security of $\mathrm{VC}$ systems is indispensable and for this, by making use of fundamental laws of quantum physics through quantumdomain communication, an unconditionally secure link for data transmission can be guaranteed [2]-[4]. More explicitly, any measurement made by an eavesdropper in entanglementbased quantum communication destroys the associated quantum entanglement and thus allows the transmitter and receiver

Copyright (c) 2017 IEEE. Personal use of this material is permitted. However, permission to use this material for any other purposes must be obtained from the IEEE by sending a request to pubs-permissions@ieee.org.

M. A. M. Izhar is with the Wireless Communication Center and Ubiquitous Broadband Access Network (U-BAN) Group, UTM Razak School of Engineering and Advanced Technology, Universiti Teknologi Malaysia, Jalan Sultan Yahya Petra, Kuala Lumpur, 54100, Malaysia. E-mail: mohdazri.k1@utm.my.

Z. Babar, S. X. Ng and L. Hanzo are with the School of Electronics and Computer Science, University of Southampton, Southampton, SO17 1BJ, United Kingdom. Email: \{zb2g10, sxn, lh\}@ecs.soton.ac.uk.

This work was supported in part by the Malaysian Ministry of Higher Education, in part by the Universiti Teknologi Malaysia, in part by the European Research Council through the Advanced Fellow Grant, in part by the Royal Society's Wolfson Research Merit Award, and in part by the Engineering and Physical Sciences Research Council under Grant EP/L018659/1. The research data for this paper is available at [http://doi.org/10.5258/SOTON/D0342]. to detect eavesdropping [2]. In this context, entanglementassisted transmission of classical information over a quantum channel would be of particular interest for high security communication applications including VCs [3], [4].

The mapping of the classical to quantum information can be implemented using the superdense (SD) coding protocol by exploiting maximally entangled states [5]. Both classical and quantum-domain channel coding aided SD protocols may be employed for protecting the information from the decoherence of quantum channels. An efficient near-capacity channel coding scheme using a classical irregular convolutional code (IRCC) and a unity rate code (URC) combined with the SD protocol was conceived in [6]. However, bit-based iterative decoding was invoked, which resulted in an inherent capacity loss [6]. In order to circumvent this capacity loss, symbolbased iterative decoding schemes were developed in [7], [8]. In particular, in [7], a serial concatenation of a convolutional code (CC) and a URC was considered while a duo-binary turbo code was employed in [8]. Both of the symbol-based coding schemes of [7] and [8] exhibited superiority over the bit-based IRCC-CC [6] and binary turbo codes, respectively. In [7], the coding parameters were optimized using extrinsic information transfer (EXIT) chart analysis and it was demonstrated that the resultant coding scheme performed within $1 \mathrm{~dB}$ from the achievable entanglement-assisted classical capacity.

The existing contributions have assumed that the classical information is uniformly distributed and memoryless, which can only be achieved by using an optimal source coding scheme exhibiting zero residual redundancy at the output of the source encoder. However, in reality, all finite-complexity and finite-delay source encoders exhibit residual redundancy in the source coded bit stream, while the natural redundancy of uncompressed sources can be much higher [9]. In the classical transmission scenario, there have been several contributions dedicated onto exploiting of a correlated source in conjunction with channel decoding which mainly relied on exploiting the source correlation using a soft-bit source decoder [10]-[16] and by modifying the channel decoding algorithm to incorporate the a priori or a posteriori source knowledge [9], [17][21]. It has been shown that the existing coding schemes of [9], [18]-[20] for example are capable of achieving near-capacity performance for transmission of classical information over a classical channel. However, there is no guarantee that these existing coding schemes can achieve the same performance in the face of the so-called quantum depolarizing channel model popularly used for modelling the quantum bit-flips and phase-flips encountered in the quantum domain owing to 
the deleterious effects of quantum de-coherence. Furthermore, they cannot be employed directly in hybrid classical-quantum communication systems, since they have to be integrated with the SD encoder before the information can be transmitted over the quantum channel. Since we invoke iterative extrinsic information exchange between the SD decoder and the channel decoder, the careful matching of these two components is vital for achieving the best possible performance of the system. Another important issue is that the bit-based coding schemes proposed in [9], [18]-[20] will require symbol-to-bit conversion from the SD decoder to the channel decoder and as detailed in [6], [7] - this will result in an inherent capacity loss, which cannot be recovered by the bit-based coding schemes of [9], [18]-[20].

Against this background, we introduce the design of nearcapacity channel coding aided SD protocol for transmission of temporally-correlated binary sources over a quantum channel. Our novel contributions can be summarized as follows:

1) We conceive a symbol-based iterative decoding scheme for the transmission of a correlated classical binary source over a quantum channel via a two-qubit SD protocol. We adopted a similar coding structure to the symbol-based coding scheme of [7] but an improved symbol-based maximum a posteriori (MAP) algorithm is conceived for the decoding of the $\mathrm{CC}$ to exploit the source correlation. Our symbol-based CC-URC-SD (SCUS) coding scheme proposed for binary Markov sources (BMS) is termed as SCUS-BMS.

2) A symbol-based EXIT chart technique is used for analyzing extrinsic information gain achieved upon exploiting the source correlation which allows us to conduct a search for the specific generator polynomial (GP) to be used by the $\mathrm{CC}$ for sources exhibiting different correlations.

3) Based on our simulation results, the proposed SCUSBMS scheme achieves performance closer to the entanglement-assisted classical capacity with respect to sources having stronger correlations. The gap to the capacity reduced from $1.01 \mathrm{~dB}$ to $0.53 \mathrm{~dB}$ for sources with correlation coefficient ${ }^{1} \rho=0$ to $\rho=0.9$, respectively.

The rest of the paper is organized as follows. In Section II, we describe the architecture of the proposed SCUS-BMS scheme and derive the entanglement-assisted classical capacity of correlated sources. Our EXIT chart analysis of the proposed decoding technique is presented along with our simulation results in Section III. Finally, our concluding remarks are provided in Section IV.

\section{SYSTEM MODEL}

We consider a first-order binary Markov source $u_{t}$, whose value depends on the previous value $u_{t-1}$ characterized by the conditional probability $\operatorname{Pr}\left\{u_{t} \mid u_{t-1}\right\}$. The two-state Markov

\footnotetext{
${ }^{1}$ The correlation coefficient represents the autocorrelation amongst the consecutive values of the same variable at different time instants. In this work, a first-order Markov chain is assumed and therefore, the correlation coefficient considered in this paper is associated with the autocorrelation between a pair of consecutive values.
}

model considered consists of four possible transitions corresponding to transition probabilities $a_{i^{\prime} i}=\operatorname{Pr}\left\{u_{t}=i \mid u_{t-1}=\right.$ $\left.i^{\prime}\right\}$, where we have $i^{\prime}, i \in\{0,1\}$ and this set of transition probabilities can be conveniently represented by the $2 \times 2$ matrix of

$$
\mathbf{A}=\left[a_{i^{\prime} i}\right]=\left[\begin{array}{cc}
a_{00}=p_{0} & a_{01}=1-p_{0} \\
a_{10}=1-p_{1} & a_{11}=p_{1}
\end{array}\right],
$$

where $p_{0}$ and $p_{1}$ are the transition probabilities associated with the transition from the Markov state " 0 " to state " 0 " and from the Markov state " 1 " to state " 1 ", respectively.

The entropy of a stationary Markov source is given by [22]

$$
H(\mathcal{U})=-\sum_{i^{\prime}, i \in\{0,1\}} \mu_{i^{\prime}} a_{i^{\prime} i} \log _{2} a_{i^{\prime} i}
$$

where $\mu_{i^{\prime}}$ is the stationary state distribution. In the case of a symmetric Markov source, where we have $p_{0}=p_{1}=p$ and $\mu_{0}=\mu_{1}=0.5$, the entropy can be simplified to $H(\mathcal{U})=$ $-p \log _{2} p-(1-p) \log _{2}(1-p)$. The correlation coefficient $\rho$ can be expressed using $p$ as $\rho=2 p-1$ [23].

\section{A. Symbol-Based CC-URC-SD for Binary Markov Sources}

We shall first describe briefly the transmission process of the SD protocol before detailing the proposed system. Again, a two-qubit SD coding protocol [5] is employed for the transmission of classical information over a quantum channel that allows a two-classical-bit message $x$ to be transmitted using a two-qubit maximally entangled Bell state $\left|\psi_{x}\right\rangle^{A B}=$ $\frac{1}{\sqrt{2}}\left(\left|0^{A} 0^{B}\right\rangle+\left|1^{A} 1^{B}\right\rangle\right)$, where $A$ and $B$ are the information qubit and the pre-shared entangled qubit, respectively. The preshared qubit is assumed to be transmitted to the receiver over a noiseless quantum channel. The SD protocol encodes the two classical bits into the corresponding quantum state, i.e., the information qubit $A$ is transformed to qubit $A^{\prime}$ which is then transmitted over a quantum channel to yield a channel-output qubit $B^{\prime}$. Finally, a symbol-by-symbol Bell basis measurement [2] is performed on the received quantum state $\left|\psi_{y}\right\rangle^{B^{\prime} B}$ to obtain a two-bit classical message $y$, which is the noisy version of the classical message $x$.

Fig. 1 illustrates the block diagram of the proposed SCUSBMS coding scheme inspired by the concept of [7] which however considered uniform and memoryless sources. In our work, a Markov source exhibiting an $N$-bit symbols $\mathbf{u}=$ $\left\{u_{1}, u_{2}, \ldots, u_{N}\right\}$ with transition probabilities of $p_{0}$ and $p_{1}$ is considered. It is worth mentioning that the Markov source is assumed to be symmetric, i.e. we have $p_{0}=p_{1}=p$ in all our simulations. The proposed coding scheme is a three-stage serially concatenated code, where we amalgamated the SD code with our URC into a single component as the inner code, while the CC acts as the outer code. The URC is employed as a precoder to the SD code in order to efficiently spread the extrinsic information without increasing the system's delay [7]. This enables the inner decoder component to reach the $(1,1)$ mutual information point in the EXIT chart, which corresponds to perfect decoding convergence to an infinitesimally low BER, as detailed in [24]. The classical source bits $\mathbf{u}$ are first encoded by a half-rate $\mathrm{CC}$ to yield the encoded symbols $\mathbf{v}=\left\{v_{1}, v_{2}, \ldots, v_{N}\right\}$. The encoded symbol-sequence 


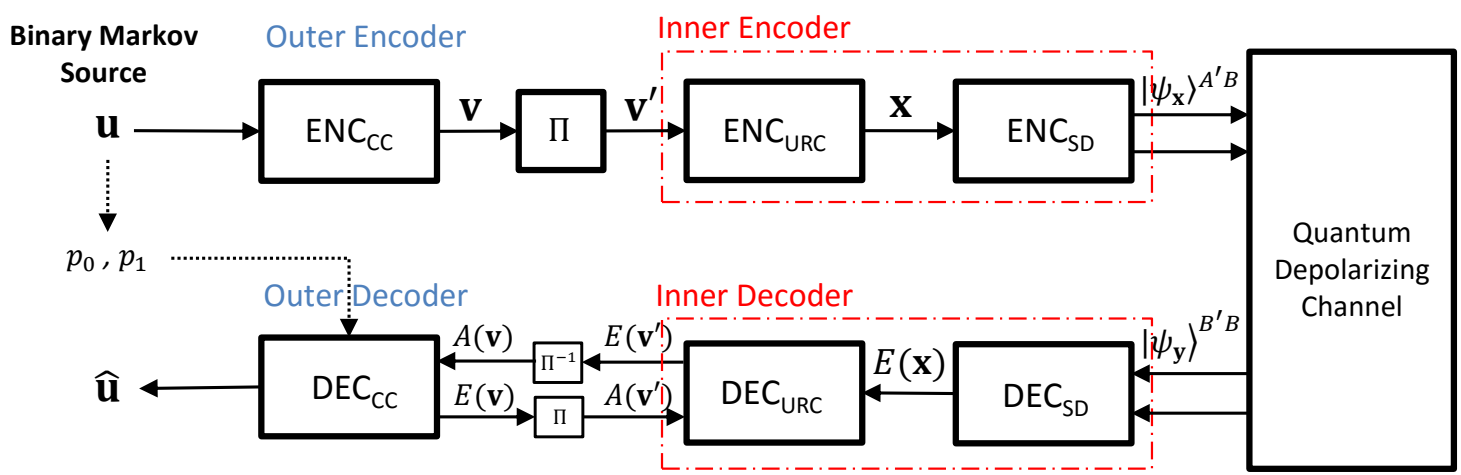

Fig. 1. Block diagram of the proposed SCUS-BMS classical-quantum communication system.

$\mathbf{v}$ is then interleaved by an $N$-symbol interleaver $\Pi$ before being encoded by a symbol-based URC have a generator polynomial $G(D)=\frac{1}{1+D}$ [25]. Then, the SD encoder is employed to transform the classical coded symbols-sequence $\mathbf{x}=\left\{x_{1}, x_{2}, \ldots, x_{N}\right\}$ into the quantum states $\left|\psi_{\mathbf{x}}\right\rangle^{A^{\prime} B}$ using the maximally entangled Bell state $\left|\psi_{\mathbf{x}}\right\rangle^{A B}$.

The SD-coded qubits $\left|\psi_{\mathbf{x}}\right\rangle^{A^{\prime} B}$ are serially transmitted over a quantum depolarizing channel. The noisy quantum channel is denoted as $\mathcal{N}^{A^{\prime} \rightarrow B^{\prime}}$ and generally, it maps an input quantum state, having a density operator of $\phi$ to a linear combination of itself with the maximally mixed state, which is given by [2], [7]

$$
\mathcal{N}_{q}(\phi)=(1-q) \phi+\frac{q}{3} \mathbf{X} \phi \mathbf{X}+\frac{q}{3} \mathbf{Y} \phi \mathbf{Y}+\frac{q}{3} \mathbf{Z} \phi \mathbf{Z},
$$

where $q$ is the depolarizing probability of the channel, while, $\mathbf{X}, \mathbf{Z}$ and $\mathbf{Y}$ are the Pauli matrices associated with a bit flip error, a phase flip error or both errors, respectively. These errors are imposed on the information qubit with a probability of $q / 3$ for each type of error.

At the receiver, a symbol-by-symbol Bell basis measurement is performed on the received quantum state $\left|\psi_{y}\right\rangle^{B^{\prime} B}$ by the SD decoder DEC $_{\text {SD }}$ Fig. 1. The soft-decision output SD decoder of [6] is employed and at the output, the extrinsic probability $E(\mathbf{x})$ corresponding to the classical transmitted symbols $\mathbf{x}$ is computed which is then passed to the URC decoder DEC $_{\text {URC. }}$ Symbol-based iterative decoding exchanging extrinsic information between $\mathrm{DEC}_{\mathrm{URC}}$ and the CC MAP decoder $\mathrm{DEC}_{\mathrm{CC}}$ is invoked, as shown in Fig. 1. The $a$ priori probability related to the $\mathrm{CC}$-coded sequence $\mathbf{v}$ and its interleaved version, $\mathbf{v}^{\prime}$ are denoted as $A(\mathbf{v})$ and $A\left(\mathbf{v}^{\prime}\right)$, respectively. Meanwhile, the extrinsic probability of the $\mathrm{CC}$-coded sequence $\mathbf{v}$ and its interleaved version, $\mathbf{v}^{\prime}$ are denoted as $E(\mathbf{v})$ and $E\left(\mathbf{v}^{\prime}\right)$, respectively. The a priori information based on the source correlation is given by the transition probabilities of $p_{0}$ as well as $p_{1}$ which are assumed to be perfectly known at the receiver by feeding it to $\mathrm{DEC}_{\mathrm{CC}}$. In order to exploit the source correlation, a modified MAP algorithm is employed at $\mathrm{DEC}_{\mathrm{CC}}$. Our modified MAP algorithm will be conceived in Section II-B. The extrinsic information exchanging process between $\mathrm{DEC}_{\mathrm{CC}}$ and $\mathrm{DEC}_{\mathrm{URC}}$ is invoked for a number of iterations and after the final iteration, hard-decision is performed based on the a posteriori probability outputs corresponding to $\mathbf{u}$ and gleaned from $\mathrm{DEC}_{\mathrm{CC}}$ for obtaining the estimated information bit-sequence $\hat{\mathbf{u}}$.

\section{B. Modified MAP Algorithm Exploiting the Source Correla- tion}

In [7], uniform and memoryless binary sources were considered and thus, the classic symbol-based MAP algorithm of [26] - which assumes dependency between the trellis states in the time-domain - was invoked for CC decoding. By contrast, we consider temporally-correlated sources and since the classic MAP algorithm is unable to exploit the source correlation, less reliable outputs may be delivered by the classic MAP algorithm, which leads to a performance degradation. Therefore, we propose to modify the classic MAP algorithm for exploiting the source correlation. Explicitly, the transition probabilities of the Markov source can be exploited by the MAP algorithm, which is achieved by incorporating the two Markov states into the CC trellis, which increases the total number of trellis states from $M$ to $2 M$.

By further developing the modified MAP algorithm of [20] originally conceived for bit-based CC MAP decoding, we can derive the modified symbol-based MAP algorithm required for our system. In relation to the algorithm of [20], our proposed algorithm is different in the following aspects: (a) we modify the symbol-based MAP algorithm, while the modification of the algorithm in [20] is performed on the bit-based MAP algorithm; (b) since we invoke symbol-based iterative decoding, the information being exchanged between the decoders is given in the form of symbol-probability and therefore, our modified algorithm is derived based on the symbol-probability-input and symbol-probability-output. On the other hand, bit-based iterative decoding is invoked in [20] and hence, the algorithm of [20] is derived based on the loglikehood ratio (LLR)-input and LLR-output.

At a timing index $t$, there are two outputs that have to be generated in our system, which are the a posteriori probability associated with the information bit $u_{t}$ defined as $\operatorname{Pr}\left\{u_{t} \mid \mathbf{y}\right\}$ and the a posteriori probability associated with the coded 
symbol $v_{t}$ defined as $\operatorname{Pr}\left\{v_{t} \mid \mathbf{y}\right\}$ where $\mathbf{y}$ is the received signal sequence. The Markovian model yields the a posteriori probability $\operatorname{Pr}\left\{u_{t}=i \mid \mathbf{y}\right\}$ associated with the state transition from $u_{t-1}=i^{\prime}$ to $u_{t}=i$ with $i^{\prime}, i \in\{0,1\}$, which is given by [20]

$$
\begin{array}{r}
\operatorname{Pr}\left\{u_{t}=i \mid \mathbf{y}\right\}=\sum_{s^{\prime}=0}^{M-1} \sum_{i^{\prime}=0}^{1} \alpha_{t-1}\left(s^{\prime}, i^{\prime}\right) \cdot \gamma_{t}\left(s^{\prime}, i^{\prime}, i\right) \\
\cdot \beta_{t}\left(L_{s}\left(s^{\prime}, i\right), i\right),
\end{array}
$$

where $\alpha_{t}$ is the forward recursive coefficient, $\beta_{t}$ is the backward recursive coefficient, $\gamma_{t}$ is the branch transition metric and $L_{s}\left(s^{\prime}, i\right)$ is a lookup table outputting the current trellis state $S_{t}=s$ corresponding to the previous trellis state $S_{t-1}=s^{\prime}$ and to the input bit $u_{t}=i$. Meanwhile, the computation of $\operatorname{Pr}\left\{v_{t}=j \mid \mathbf{y}\right\}$ with $j \in\{0,1,2,3\}$ is given by

$$
\begin{aligned}
\operatorname{Pr}\left\{v_{t}=L_{j}\left(s^{\prime}, i\right) \mid \mathbf{y}\right\}= & \sum_{s^{\prime}=0}^{M-1} \sum_{i^{\prime}=0}^{1} \sum_{i=0}^{1} \alpha_{t-1}\left(s^{\prime}, i^{\prime}\right) \\
& \cdot \gamma_{t}\left(s^{\prime}, i^{\prime}, i\right) \cdot \beta_{t}\left(L_{s}\left(s^{\prime}, i\right), i\right),
\end{aligned}
$$

where $L_{j}\left(s^{\prime}, i\right)$ represents the lookup table outputting the coded symbol $v_{t}=j$ corresponding to the previous trellis state $S_{t-1}=s^{\prime}$ and to the input bit $u_{t}=i$. The forward recursive coefficient $\alpha_{t}$ and the backward recursive coefficient $\beta_{t}$ are formulated as [20]:

$$
\begin{aligned}
\alpha_{t}(s, i) & =\operatorname{Pr}\left\{S_{t}=s, u_{t}=i \mid \mathbf{y}_{\leq t}\right\} \\
& =\sum_{i^{\prime}=0}^{1} \alpha_{t-1}\left(L_{s^{\prime}}(s, i), i^{\prime}\right) \cdot \gamma_{t}\left(L_{s^{\prime}}(s, i), i^{\prime}, i\right)
\end{aligned}
$$

and

$$
\begin{aligned}
\beta_{t-1}\left(s^{\prime}, i^{\prime}\right) & =\operatorname{Pr}\left\{S_{t-1}=s^{\prime}, u_{t-1}=i^{\prime} \mid \mathbf{y}_{>t}\right\} \\
& =\sum_{i=0}^{1} \beta_{t}\left(L_{s}\left(s^{\prime}, i\right), i\right) \cdot \gamma_{t}\left(s^{\prime}, i^{\prime}, i\right),
\end{aligned}
$$

respectively, where $L_{s^{\prime}}(s, i)$ represents the lookup table outputting the previous trellis state $S_{t-1}=s^{\prime}$ corresponding to $S_{t}=s$ and $u_{t}=i$. The boundary conditions for $\alpha_{t}$ at $t=0$ are given by $\alpha_{0}(0, i)=\mu_{i}$ and $\alpha_{0}(s, i)=0$ for all $s \neq 0$, while the boundary condition for $\beta_{t}$ at $t=N$ is given by $\beta_{N}(s, i)=\frac{1}{2 M}$ for all $s$. The branch transition metric $\gamma_{t}$ is defined as [20]

$$
\begin{aligned}
\gamma_{t}\left(s^{\prime}, i^{\prime}, i\right)= & \operatorname{Pr}\left\{S_{t}=s, u_{t}=i, y_{t} \mid S_{t-1}=s^{\prime}, u_{t-1}=i^{\prime}\right\}, \\
= & \operatorname{Pr}\left\{S_{t}=s, u_{t}=i \mid S_{t-1}=s^{\prime}, u_{t-1}=i^{\prime}\right\} \\
& \cdot \operatorname{Pr}\left\{y_{t} \mid S_{t}=s, u_{t}=i\right\} .
\end{aligned}
$$

The joint probability $\operatorname{Pr}\left\{S_{t}=s, u_{t}=i \mid S_{t-1}=s^{\prime}, u_{t-1}=\right.$ $\left.i^{\prime}\right\}$ in Eq. (8) is influenced by two factors, namely by the transition probability of the Markov source and by the state transition from $s^{\prime}$ to $s$ that associated to the input bit $u_{t}=i$ and correspondingly, the output symbol $v_{t}=j$. Therefore, Eq. (8) can be approximated as

$$
\begin{aligned}
\gamma_{t}\left(s^{\prime}, i^{\prime}, i\right) \approx & \operatorname{Pr}\left\{u_{t}=i \mid u_{t-1}=i^{\prime}\right\} \\
& \cdot \operatorname{Pr}\left\{u_{t}=i\right\} \cdot \operatorname{Pr}\left\{v_{t}=j\right\} \\
& \cdot \operatorname{Pr}\left\{y_{t} \mid S_{t}=s, u_{t}=i\right\} \\
= & a_{i^{\prime} i} \cdot \operatorname{Pr}\left\{u_{t}=i\right\} \\
& \cdot \operatorname{Pr}\left\{v_{t}=j\right\} \cdot \operatorname{Pr}\left\{y_{t} \mid S_{t}=s, u_{t}=i\right\} \\
= & a_{i^{\prime} i} \cdot \operatorname{Pr}\left\{u_{t}=i\right\} \cdot \operatorname{Pr}\left\{v_{t}=j\right\} \cdot \operatorname{Pr}\left\{y_{t} \mid x_{t}\right\},(9)
\end{aligned}
$$

where $\operatorname{Pr}\left\{u_{t}=i\right\}$ and $\operatorname{Pr}\left\{v_{t}=j\right\}$ are the a priori probabilities corresponding to $u_{t}=i$ and $v_{t}=j$, respectively and $a_{i^{\prime} i}$ is the transition probability defined in Eq. (1). From Fig. 1, we can see that only the a priori probability associated with $v_{t}$ inputs to $\mathrm{DEC}_{\mathrm{CC}}$ and since there is no a priori information associated with $u_{t}$, we have $\operatorname{Pr}\left\{u_{t}=0\right\}=\operatorname{Pr}\left\{u_{t}=1\right\}=0.5$. It should be noted also that the a priori symbol probability $A\left(v_{t}\right)$ in Fig. 1 has included the information from the channel's transition probability $\operatorname{Pr}\left\{y_{t} \mid x_{t}\right\}$ (given by $E\left(x_{t}\right)^{2}$ ) and thus, Eq. (9) can be expressed as

$$
\gamma_{t}\left(s^{\prime}, i^{\prime}, i\right)=0.5 \cdot a_{i^{\prime} i} \cdot A\left(v_{t}=L_{j}\left(s^{\prime}, i\right)\right) .
$$

Once the a posteriori information $\operatorname{Pr}\left\{v_{t} \mid \mathbf{y}\right\}$ in Eq. (5) has been computed, the extrinsic information $E\left(v_{t}\right)$ can then be obtained by removing the a priori information $A\left(v_{t}\right)$ from the resulted a posteriori information $\operatorname{Pr}\left\{v_{t} \mid \mathbf{y}\right\}$.

\section{Entangelmend-Assisted Classical Channel Capacity for Binary Markov Sources}

The entanglement-assisted classical capacity $C_{2 \mathrm{SD}}$ for transmission of classical information over a quantum depolarizing channel using a two-qubit SD protocol is given by [5]

$$
C_{2 \mathrm{SD}}=2+(1-q) \log _{2}(1-q)+q \log _{2}(q / 3) \quad \text { cbits } / \mathrm{use}^{3},
$$

when the pre-shared qubit is assumed to be perfectly received.

Fig. 2 illustrates the capacity curve of $C_{2 \mathrm{SD}}$ against different depolarizing probability values $q$. The effective throughput of our proposed system is given by $\eta=H(\mathcal{U}) \cdot R_{\mathrm{c}} \cdot R_{\mathrm{SD}}$, where $R_{\mathrm{c}}$ and $R_{\mathrm{SD}}$ are the code rates of channel coding and of the SD protocol, respectively. In the considered system, we have $R_{\mathrm{c}}=0.5$ and $R_{\mathrm{SD}}=2$ cbits/use, and hence, $\eta=H(\mathcal{U})$. The entropy $H(\mathcal{U})$ is dependent on the strength of the source correlation and can be quantified using Eq. (2). Based on Eq. (2), the entropy of symmetric Markov sources associated with $\rho=$ $\{0,0.2,0.4,0.6,0.8,0.9\}$ are $\{1,0.97,0.88,0.72,0.47,0.29\}$ bit, respectively. The maximum limit for the depolarizing probability $q_{\text {lim }}$ to guarantee a reliable transmission can be determined from the capacity curve of Fig. 2. It is shown that for sources having a correlation with transition probability $p$ close to 1 , the $q_{\lim }$ value becomes higher, hence achieving reliable communications over a more hostile quantum channel.

\footnotetext{
${ }^{2}$ In [7], the computation of the extrinsic output $E\left(x_{t}\right)$ from the SD decoder is given by $E\left(x_{t}\right) \approx \operatorname{Pr}\left\{y_{t} \mid x_{t}\right\}$.

${ }^{3}$ Classical bits per channel use.
} 


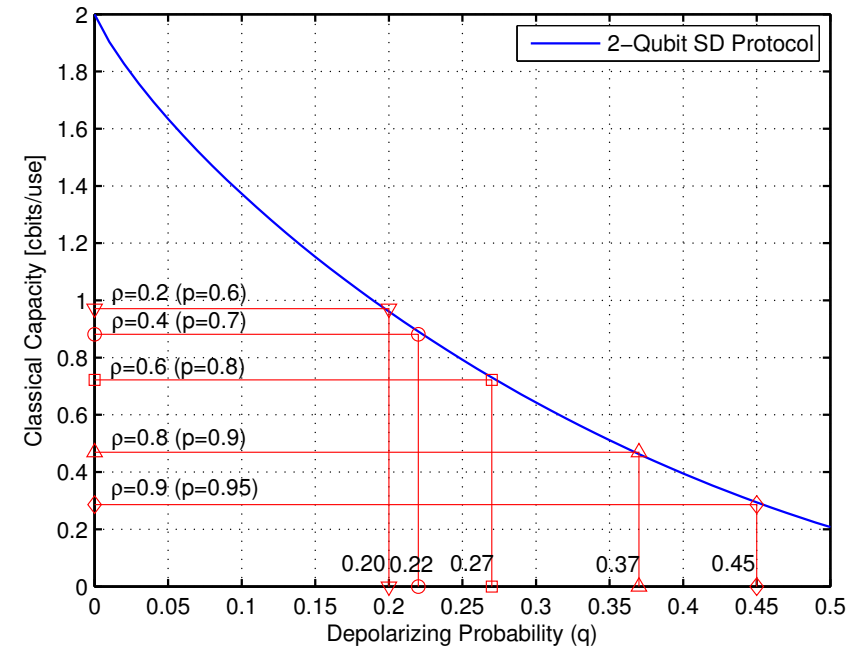

Fig. 2. Classical information rate against quantum depolarizing probability for a two-qubit SD protocol. For memoryless sources, the effective throughput is $1 \mathrm{cbit} / \mathrm{use}$, which corresponds to $q=0.19$.

\section{NUMERICAL RESUlts}

In this section, we first analyze the convergence of the proposed decoding technique using EXIT charts. The impact of Markov sources associated with different correlations on the extrinsic mutual information (MI) of $\mathrm{DEC}_{\mathrm{CC}}$ will be examined. Then the bit error rate (BER) performance of the proposed scheme will be evaluated and compared to other schemes, including a turbo coding-based SD scheme.

\section{A. EXIT Chart Analysis}

EXIT charts are capable of visualizing the convergence of an iterative decoding scheme [27]. The non-binary EXIT chart approach of [28] is employed for analyzing the convergence behaviour of our symbol-based iterative decoding scheme designed for correlated sources. We evaluate the EXIT characteristics of $\mathrm{DEC}_{\mathrm{CC}}$ of Fig. 1 as the outer component decoder and the amalgamated of $\mathrm{DEC}_{\mathrm{SD}}+\mathrm{DEC}_{\mathrm{URC}}$ as the inner component decoder. In contrast to the outer decoder of [7], the modified MAP algorithm employed in our $\mathrm{DEC}_{\mathrm{CC}}$ will affect the EXIT curve of $\mathrm{DEC}_{\mathrm{CC}}$, depending on the source correlation. Thus, the EXIT function $T_{\text {out }}($.$) of our outer$ decoder is given by

$$
I_{\mathrm{E}, \text { out }}=T_{\text {out }}\left(I_{\mathrm{A}, \text { out }}, \rho\right)
$$

where $I_{\mathrm{E}, \text { out }}$ and $I_{\mathrm{A} \text {,out }}$ are the average extrinsic and a priori mutual information corresponding to $\mathbf{v}$ of the outer decoder, respectively. Meanwhile, the EXIT function $T_{\text {in }}($.$) of our inner$ decoder is the same as that of the inner decoder of [7], which is given by

$$
I_{\mathrm{E}, \text { in }}=T_{\mathrm{in}}\left(I_{\mathrm{A}, \text { in }}, q\right),
$$

where $I_{\mathrm{E}, \text { in }}$ and $I_{\mathrm{A} \text {,in }}$ are the average extrinsic and a priori mutual information corresponding to $\mathbf{v}^{\prime}$ for the inner decoder, respectively. The function $T_{\text {in }}($.$) does not depend on the$ source correlation coefficient $\rho$ but rather it depends on the depolarizing probability $q$, since it is the inner decoder, which is fed by the channel and not the source. For non-binary

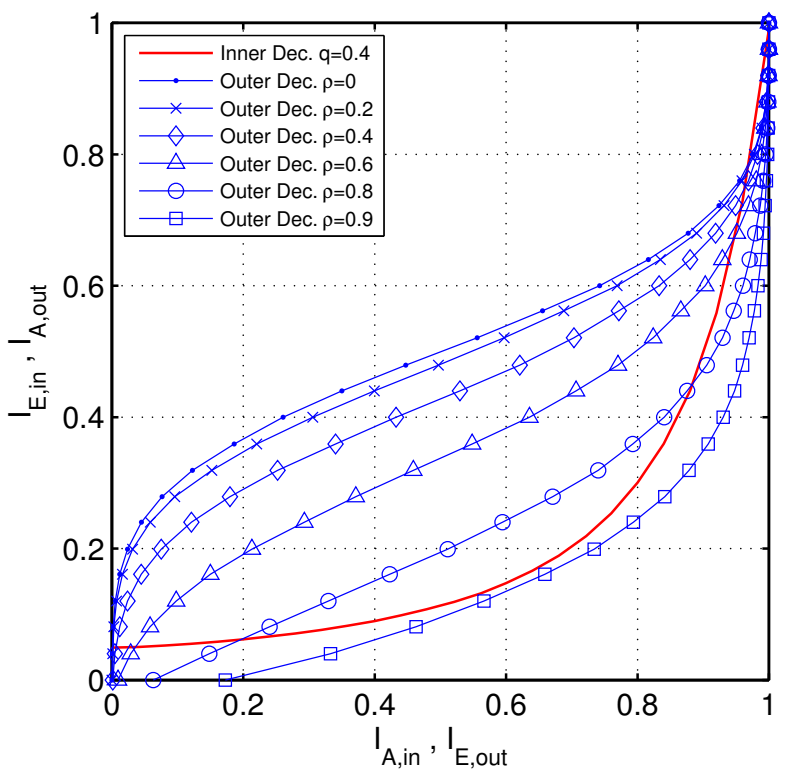

Fig. 3. EXIT characteristics of the inner decoder at $q=0.4$ and outer decoder for sources with $\rho=\{0,0.2,0.4,0.6,0.8,0.9\}$. The interleaver length for $\Pi$ was set to 12,000 symbols and the memory- 4 non-systematic CC with GP $\left(g_{1}, g_{2}\right)=(25,21)_{8}$ (in octal notation) was employed for $\mathrm{DEC}_{\mathrm{CC}}$.

symbols, the MI value can be in excess of one bit and in our case, the maximum value of the MI is two bits. However, for simplicity, all the MIs considered in this work have been normalized to unity.

Fig. 3 shows the different EXIT characteristics of the outer decoder for sources having different correlation coefficients. As expected, a higher $I_{\mathrm{E}, \text { out }}$ gain can be achieved over the memoryless sources in the case of higher source correlation ${ }^{4}$. This $I_{\mathrm{E}, \text { out }}$ improvement would help achieve perfect decoding convergence threshold at higher depolarizing probability values. For example, as illustrated in Fig. 3, due to the intersections between the inner decoder and outer decoder EXIT curves for $\rho=\{0,0.2,0.4,0.6,0.8\}$, decoding convergence to a vanishingly low BER cannot be achieved and a high BER can be expected at $q=0.4$. However, a marginally open tunnel can be seen between the EXIT curves of the inner decoder and outer decoder at the source correlation of $\rho=0.9$. Therefore, convergence to an infinitesimally low BER at $q=0.4$ can be expected for sources associated with $\rho=0.9$.

We conducted an exhaustive search using EXIT charts to find the specific GP for the outer code that can achieve nearoptimal performance for all the evaluated source correlations. We tested all possible memory-2, memory- 3 and memory- 4 non-systematic CCs that can have a good EXIT-curve matching with the inner decoder at a relatively high $q$ value. As noted from Eq. (12), the EXIT curve for the outer decoder depends on $\rho$ and since the correlation coefficient $\rho$ of the source can be any decimal number between 0 and 1 an, it may not be possible to practically find the best outer code for every $\rho$ value. Therefore, we only evaluated certain values of $\rho$, which are

\footnotetext{
${ }^{4}$ It was found that the EXIT curve of memoryless sources relying on the original MAP algorithm is identical to the EXIT curve of the modified MAP algorithm for sources associated with $\rho=0$.
} 


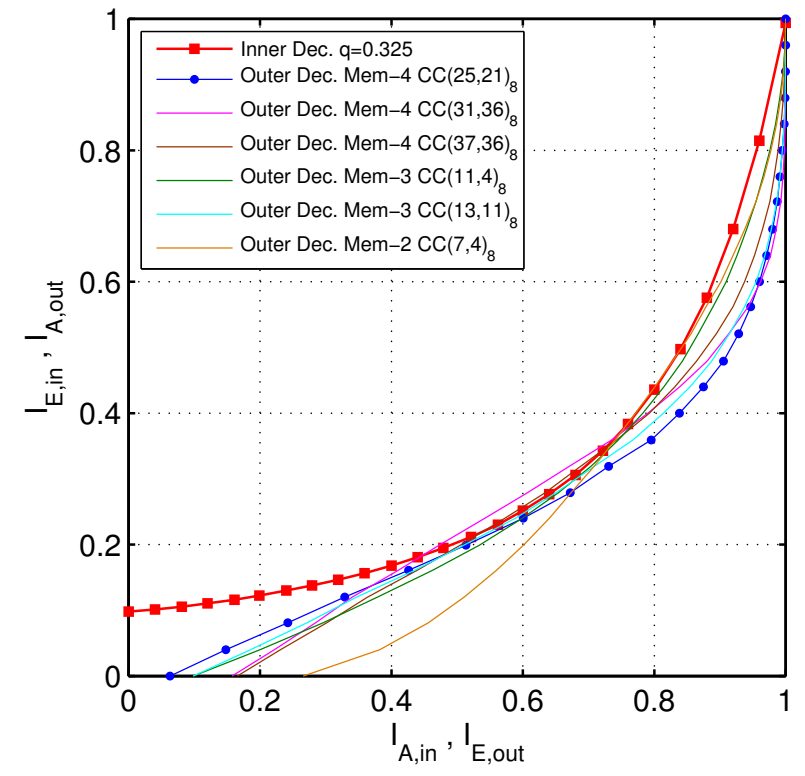

Fig. 4. EXIT characteristics of the inner decoder at $q=0.325$ and various types of non-systematic CCs employed as the outer component of the proposed system for sources with $\rho=0.8$. The memory- 4 non-systematic CC with GP $(25,21)_{8}$ has the best EXIT curve matching with the inner decoder.

$\rho=\{0,0.2,0.4,0.6,0.8,0.9\}$ and searched for an outer code that can generally achieve near-optimal performance for all the evaluated $\rho$ values. Based on our exhaustive search using EXIT charts, we found that the non-systematic CC associated with the octally represented GP of $(25,21)_{8}$ can achieve the best or near best performance for all the evaluated source correlations. For example, Fig. 4 illustrates the EXIT curves of some of these CCs when utilizing a source correlation of $\rho=0.8$, including the memory-4 CC with GP $(31,36)_{8}$, which was shown to be the optimal GP in [7] for memoryless sources. As seen from the figure, the memory- 4 non-systematic $\mathrm{CC}$ with GP $(25,21)_{8}$ has the best EXIT curve matching with the inner decoder at $q=0.325$ as the EXIT curves for the other outer decoders intersected with the inner decoder's curve. However, this cannot be guaranteed for other sources with different source correlation. In such cases, we found that employing the CC using GP $(25,21)_{8}$ can still outperform the CC in conjunction with GP $(31,36)_{8}$ [7] and achieve a performance near to the optimal.

\section{B. BER Performance Evaluation}

A series of computer simulations was conducted to evaluate the proposed SCUS-BMS scheme. We ran simulations using a frame length of $N=12,000$ bits and considered symmetric Markov sources associated with $\rho=\{0,0.2,0.4,0.6,0.8,0.9\}$. The number of decoding iterations was set to 25 and in order to ensure a good reliability of the data, at least 1000 frames were simulated for each $q$ value. Based on the EXIT chart analysis of the previous section, the memory- 4 non-systematic $\mathrm{CC}$ with GP $(25,21)_{8}$ was employed for DEC $_{\mathrm{CC}}$.

The classical BER performance of the proposed SCUSBMS scheme relying on source correlations of $\rho=$ $\{0,0.2,0.4,0.6,0.8,0.9\}$ is depicted in Fig. 5. BER perfor-

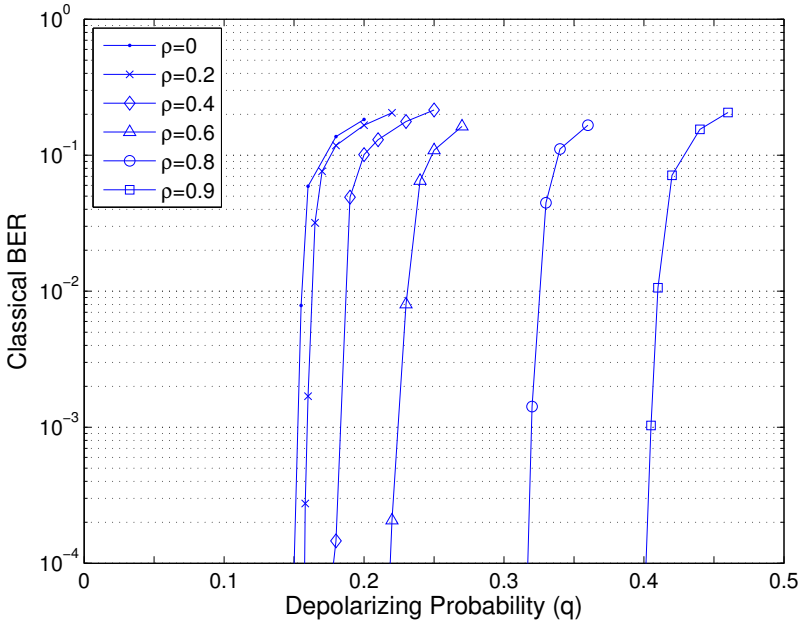

Fig. 5. Classical BER performance of the proposed SCUS-BMS scheme when employing the memory-4 non-systematic CC with GP $(25,21)_{8}$ as the outer code for sources with $\rho=\{0,0.2,0.4,0.6,0.8,0.9\}$.

mance improvements can be observed, when utilizing sources with stronger correlation using the proposed SCUS-BMS scheme. The BER performance exhibits consistency with the EXIT chart predictions. For example, for sources associated with $\rho=0.9$, a BER of $10^{-4}$ is achieved at $q \approx 0.4$, which is the convergence threshold determined from the EXIT chart of Fig. 3.

The correlation coefficient versus depolarizing probability is plotted in Fig. 6 at a BER of $10^{-4}$ and compared to other schemes, including the maximum error-free capacity limit. We first compare the proposed SCUS-BMS scheme to the same SCUS-BMS arrangement, but employing the memory-4 CC using GP $(31,36)_{8}$ as the outer code, which was shown to be the best code for memoryless sources in [7]. There is almost no difference in performance between the SCUSBMS using $\mathrm{CC}(25,21)_{8}$ and $\mathrm{CC}(31,36)_{8}$ for sources having zero or low correlation. However, for high source correlation scenarios (i.e., $\rho \geq 0.6$ ), significant performance gains can be observed for SCUS-BMS using the proposed CC $(25,21)_{8}$ over $\mathrm{CC}(31,36)_{8}$.

The proposed SCUS-BMS scheme is then benchmarked against two other bit-based coding schemes. The first coding scheme is the bit-based CC-URC-SD for BMS (BCUS-BMS) coding scheme, which is similar to the SCUS-BMS scheme, but performs iterative decoding at the bit-level instead of symbol-level ${ }^{5}$. The second coding scheme is based on the concatenation of a turbo code (TC) [29], [30] and an SD protocol which is defined as the TC-SD-BMS coding scheme. It was shown in [9] that employing a systematic CC using GP $(35,23)_{8}$ as the component code for a turbo code achieves optimal performance in most cases for the transmission of correlated information over a classical channel. Therefore, we opted for employing the same systematic CC using GP $(35,23)_{8}$ as the component code for the turbo code used in the

${ }^{5}$ BCUS-BMS has the same structure as SCUS-BMS, but the symbol interleaver/deinterleaver is replaced by a bit interleaver/deinterleaver and where necessary, symbol-to-bit and bit-to-symbol converters are employed in the system. 


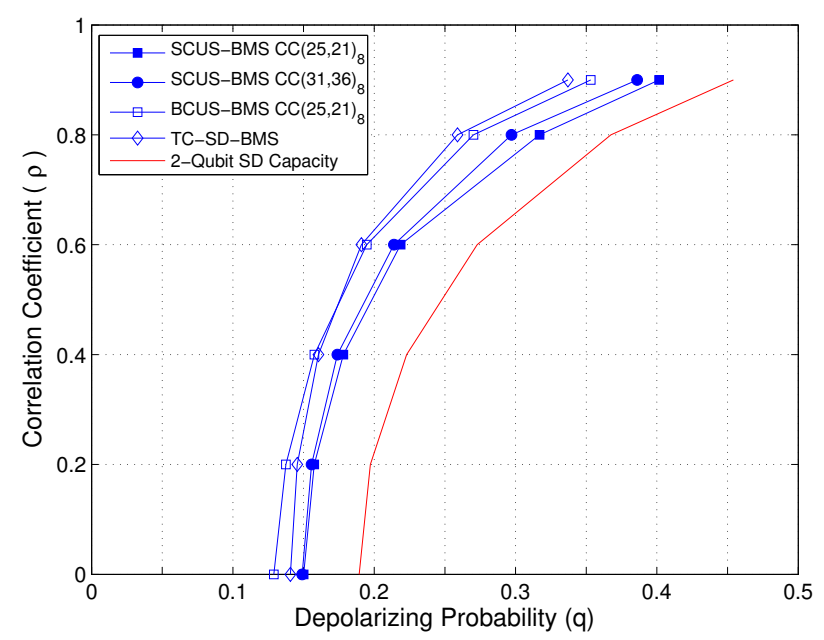

Fig. 6. Comparison in BER performance at $10^{-4}$ between the proposed SCUS-BMS, BCUS-BMS and TC-SD-BMS

TABLE I

GAP TO THE THEORETICAL LIMIT

\begin{tabular}{|c|c|c|c|}
\hline \multirow{2}{*}{$\rho$} & \multicolumn{3}{|c|}{ Gap (dB) } \\
\cline { 2 - 4 } & SCUS-BMS & BCUS-BMS & TC-SD-BMS \\
\hline 0.0 & 1.01 & 1.67 & 1.29 \\
\hline 0.2 & 0.98 & 1.57 & 1.32 \\
\hline 0.4 & 0.97 & 1.50 & 1.43 \\
\hline 0.6 & 0.96 & 1.47 & 1.55 \\
\hline 0.8 & 0.64 & 1.33 & 1.52 \\
\hline 0.9 & 0.53 & 1.09 & 1.30 \\
\hline
\end{tabular}

TC-SD-BMS coding scheme. The performance of the BCUSBMS and TC-SD-BMS schemes is compared to the proposed SCUS-BMS scheme in Fig. 6. More explicitly, the proposed SCUS-BMS scheme outperforms both the BCUS-BMS and the TC-SD-BMS schemes for all sources regardless of the $\rho$ values.

The curve associated with the capacity limit is also plotted in Fig. 6 based on the $q_{\text {lim }}$ given in Fig. 2. The gap with respect to the theoretical limit in $\mathrm{dB}$ is given by $10 \log \left(q_{\lim } / q_{10^{-4}}\right)$ [6] where $q_{10^{-4}}$ is the depolarizing probability value at BER of $10^{-4}$. Based on the performance seen in Fig. 6, the gap with respect to the theoretical limit for the SCUS-BMS, BCUSBMS and TC-SD-BMS schemes for sources associated with $\rho=\{0,0.2,0.4,0.6,0.8,0.9\}$ is summarized in Table I. The proposed SCUS-BMS has the performance which is the closest to the capacity namely within $1.01 \mathrm{~dB}$ for all the evaluated sources. A significant reduction of the gap to the capacity can be observed for sources with $\rho \geq 0.6$, which reduced from $0.96 \mathrm{~dB}$ for $\rho=0.6$ to only $0.53 \mathrm{~dB}$ for $\rho=0.9$. Therefore, a near-capacity performance can be achieved by using the proposed SCUS-BMS scheme. Furthermore, the gap approaches zero for even higher source correlations.

Despite the excellent performance achieved for classical channels in [9] using TC relying on two systematic CCs having GP $(35,23)_{8}$, the performance of the same coding scheme aided by the SD protocol for quantum transmission is not as good as that of its classical counterpart. For example, the gap to the theoretical limit for sources having $\rho=0.6$ $(q=0.8)$ transmitted over the classical additive white Gaus- sian noise (AWGN) and Rayleigh fading channels are $0.94 \mathrm{~dB}$ and $1.08 \mathrm{~dB}$, respectively ${ }^{6}$ but the gap to the theoretical limit of the same TC assisted by the SD protocol for transmission over the quantum depolarizing channels is $1.43 \mathrm{~dB}$. Meanwhile, our proposed SCUS-BMS scheme achieves a performance within $0.96 \mathrm{~dB}$ from the theoretical limit for the same scenario. The performance advantage achieved by using our SCUS-BMS compared to the BCUS-BMS and TC-SD-BMS schemes is mainly due to the symbol-based iterative decoding invoked in SCUS-BMS. Furthermore, it is a benefit of our EXIT-chart aided design procedure used for finding the best outer code that can be closely matched with the inner code, i.e., URC+SD in SCUS-BMS.

\section{CONCLUSIONS}

In this paper, we have proposed a symbol-based iterative decoding scheme for the transmission of correlated classical binary source signals over a quantum channel using a threestage serial concatenation of a CC, a URC and a two-qubit SD scheme. A first-order Markov chain was assumed for modeling the correlated sources and a modified symbol-based MAP algorithm was conceived for CC decoding in order to exploit the source correlation. The EXIT chart results demonstrated that beneficial mutual information gains can be achieved upon exploiting the source correlation, where the gain achieved depends on the source correlation. Based on our exhaustive search procedure using EXIT charts, it was found that the non-systematic CC using GP $(25,21)_{8}$ achieves the best or nearly the best performance for all different source correlations considered. We also evaluated the BER performance of the proposed SCUS-BMS scheme and demonstrated the superiority of our SCUS-BMS over all the benchmark schemes including the TC-based scheme. It was shown that the proposed SCUS-BMS scheme operates within $0.53 \mathrm{~dB}$ from the capacity limit for a source correlation of $\rho=0.9$.

\section{REFERENCES}

[1] P. Papadimitratos, L. Buttyan, T. Holczer, E. Schoch, J. Freudiger, M. Raya, Z. Ma, F. Kargl, A. Kung, and J. P. Hubaux, "Secure vehicular communication systems: design and architecture," IEEE Commun. Mag., vol. 46, no. 11, pp. 100-109, Nov. 2008.

[2] M. A. Nielsen and I. L. Chuang, Quantum Computation and Quantum Information: 10th Anniversary Edition, 10th ed. New York, NY, USA: Cambridge University Press, 2011.

[3] T. S. Lin, C. H. Chien, T. H. Chang, and S. Y. Kuo, "Quantum signature scheme for vehicular networks using entangled states," in 2011 Carnahan Conf. Security Technology, Oct. 2011, pp. 1-6.

[4] R. Malaney, "The quantum car," IEEE Wireless Commun. Lett., vol. 5, no. 6, pp. 624-627, Dec. 2016.

[5] C. H. Bennett and S. J. Wiesner, "Communication via one- and two-particle operators on Einstein-Podolsky-Rosen states," Phys. Rev. Lett., vol. 69, pp. 2881-2884, Nov 1992. [Online]. Available: http://link.aps.org/doi/10.1103/PhysRevLett.69.2881

[6] Z. Babar, S. X. Ng, and L. Hanzo, "Near-capacity code design for entanglement-assisted classical communication over quantum depolarizing channels," IEEE Trans. Commun., vol. 61, no. 12, pp. 4801-4807, Dec. 2013.

[7] — "EXIT-chart aided code design for symbol-based entanglementassisted classical communication over quantum channels," in 2014 IEEE 80th Veh. Tech. Conf. (VTC2014-Fall), Sep. 2014, pp. 1-5.

${ }^{6}$ Results from Table I of [9]. 
[8] C. Boyd, R. A. Pitaval, U. Parts, and O. Tirkkonen, "Non-binary classical error-correcting codes for quantum communication," in 2015 IEEE Int. Conf. Communications (ICC), Jun. 2015, pp. 4060-4065.

[9] G. Zhu and F. Alajaji, "Joint source-channel turbo coding for binary Markov sources," IEEE Trans. Wireless Commun., vol. 5, no. 5, pp 1065-1075, May 2006.

[10] N. Görtz, "On the iterative approximation of optimal joint sourcechannel decoding," IEEE J. Select. Areas Commun., vol. 19, no. 9, pp. 1662-1670, Sep. 2001.

[11] M. Adrat, P. Vary, and J. Spittka, "Iterative source-channel decoder using extrinsic information from softbit-source decoding," in Proc. of IEEE Int. Conf. Acoustics, Speech and Signal Processing, Utah, USA, May 2001, pp. 2653-2656.

[12] M. Adrat and P. Vary, "Iterative source-channel decoding: Improved system design using EXIT charts," EURASIP J. Appl. Signal Process., vol. 2005, no. 6, pp. 928-941, May 2005.

[13] J. Kliewer, N. Görtz, and A. Mertins, "Iterative source-channel decoding with Markov random field source models," IEEE Trans. Signal Processing, vol. 54, no. 10, pp. 3688-3701, Oct. 2006.

[14] R. Thobaben and J. Kliewer, "An efficient variable-length code construction for iterative source-channel decoding," IEEE Trans. Commun., vol. 57, no. 7, pp. 2005-2013, Jul. 2009.

[15] Nasruminallah and L. Hanzo, "EXIT-chart optimized short block codes for iterative joint source and channel decoding in H.264 video telephony," IEEE Trans. Veh. Technol., vol. 58, no. 8, pp. 4306-4315, Oct. 2009.

[16] Y. Huo, C. Zhu, and L. Hanzo, "Spatio-temporal iterative source-channel decoding aided video transmission," IEEE Trans. Veh. Technol., vol. 62, no. 4, pp. 1597-1609, May 2013.

[17] J. Hagenauer, "Source-controlled channel decoding," IEEE Trans. Commun., vol. 43, no. 9, pp. 2449-2457, Sep. 1995.

[18] J. Garcia-Frias and J. D. Villasenor, "Combining hidden Markov source models and parallel concatenated codes," IEEE Commun. Lett., vol. 1, no. 4, pp. 111-113, Jul. 1997.

[19] - "Joint turbo decoding and estimation of hidden Markov sources," IEEE J. Select. Areas Commun., vol. 19, no. 9, pp. 1671-1679, Sep. 2001.

[20] X. Zhou, K. Anwar, and T. Matsumoto, "Serially concatenated joint source-channel coding for binary Markov sources," in 6th Int. ICST Conf. Communications and Networking (CHINACOM), Harbin, China, 2011.

[21] M. A. Mohd Izhar, N. Fisal, X. Zhou, K. Anwar, and T. Matsumoto, "Exploitation of 2D binary source correlation using turbo block codes with fine-tuning," EURASIP J. Wirel. Commun. Netw., vol. 2013, no. 1, p. 89, Mar. 2013.

[22] T. M. Cover and J. A. Thomas, Elements of Information Theory 2nd Edition. USA: John Wiley and Sons, 2006.

[23] A. J. Aljohani, S. X. Ng, and L. Hanzo, "TTCM-aided rate-adaptive distributed source coding for Rayleigh fading channels," IEEE Trans. Veh. Technol., vol. 63, no. 3, pp. 1126-1134, Mar. 2014.

[24] K. R. Narayanan, "Effect of precoding on the convergence of turbo equalization for partial response channels," IEEE J. Select. Areas Commun., vol. 19, no. 4, pp. 686-698, Apr. 2001.

[25] L. Hanzo, T. H. Liew, B. L. Yeap, R. Y. S. Tee, and S. X. Ng, Turbo Coding, Turbo Equalisation and Space-Time Coding: EXIT-Chart-Aided Near-Capacity Designs for Wireless Channels, 2nd Edition. New York, NY, USA: Wiley-IEEE Press, 2011.

[26] L. Bahl, J. Cocke, F. Jelinek, and J. Raviv, "Optimal decoding of linear codes for minimizing symbol error rates (corresp.)," IEEE Trans. Inform. Theory, vol. 20, no. 2, pp. 284-287, Mar. 1974.

[27] S. ten Brink, "Convergence behavior of iteratively decoded parallel concatenated codes," IEEE Trans. Commun., vol. 49, no. 10, pp. 17271737, Oct. 2001.

[28] J. Kliewer, S. X. Ng, and L. Hanzo, "Efficient computation of EXIT functions for nonbinary iterative decoding," IEEE Trans. Commun., vol. 54, no. 12, pp. 2133-2136, Dec. 2006.

[29] C. Berrou, A. Glavieux, and P. Thitimajshima, "Near shannon limit error-correcting coding and decoding: Turbo-codes," in IEEE Int. Conf. Communications (ICC), Geneva, Switzerland, May 1993, pp. 10641070.

[30] C. Berrou and A. Glavieux, "Near optimum error correcting coding and decoding: turbo-codes," IEEE Trans. Commun., vol. 44, no. 10, pp. 1261-1271, Oct. 1996. 


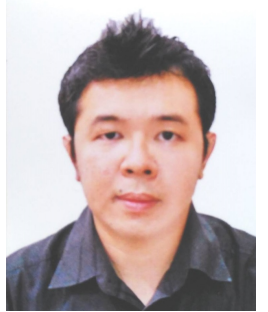

Mohd Azri Mohd Izhar received his M.Eng. degree (First class) in electrical engineering (communications) from the University of Sheffield, U.K. in 2008 and $\mathrm{Ph} . \mathrm{D}$. degree in electrical engineering from the Universiti Teknologi Malaysia (UTM), Malaysia in 2014. Since 2014, He has been a senior lecturer with the UTM Kuala Lumpur campus. On leave from the UTM, he was visiting the Southampton Wireless Group, University of Southampton, U.K. for 2 years in 2015 .

His current research interests include channel coding, coding theory, joint source-channel coding, cooperative communications, cognitive radio and quantum error correction codes.

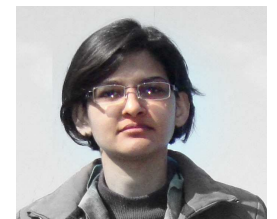

Zunaira Babar received her B.Eng. degree in electrical engineering from the National University of Science \& Technology (NUST), Islamabad, Pakistan, in 2008, and the M.Sc. degree (Distinction) and the Ph.D degree in wireless communications from the University of Southampton, UK, in 2011 and 2015 , respectively. She is currently working as a Research Fellow in the Southampton Wireless group at the University of Southampton.

Her research interests include quantum error correction codes, channel coding, coded modulation, iterative detection and cooperative communications.

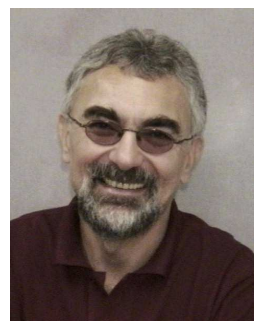

Lajos Hanzo (http://www-mobile.ecs.soton.ac.uk) FREng, FIEEE, FIET, Fellow of EURASIP, DSc received his degree in electronics in 1976 and his doctorate in 1983. In 2009 he was awarded an honorary doctorate by the Technical University of Budapest and in 2015 by the University of Edinburgh. In 2016 he was admitted to the Hungarian Academy of Science. During his 40 -year career in telecommunications he has held various research and academic posts in Hungary, Germany and the UK. Since 1986 he has been with the School of Electronics and Computer Science, University of Southampton, UK, where he holds the chair in telecommunications. He has successfully supervised 111 $\mathrm{PhD}$ students, co-authored 18 John Wiley/IEEE Press books on mobile radio communications totalling in excess of 10000 pages, published 1701 research contributions at IEEE Xplore, acted both as TPC and General Chair of IEEE conferences, presented keynote lectures and has been awarded a number of distinctions. Currently he is directing a 60 -strong academic research team, working on a range of research projects in the field of wireless multimedia communications sponsored by industry, the Engineering and Physical Sciences Research Council (EPSRC) UK, the European Research Council's Advanced Fellow Grant and the Royal Society's Wolfson Research Merit Award.

$\mathrm{He}$ is an enthusiastic supporter of industrial and academic liaison and he offers a range of industrial courses. He is also a Governor of the IEEE VTS. During 2008 - 2012 he was the Editor-in-Chief of the IEEE Press and a Chaired Professor also at Tsinghua University, Beijing. For further information on research in progress and associated publications please refer to http://wwwmobile.ecs.soton.ac.uk Lajos has $30000+$ citations and an $\mathrm{H}$-index of 70.

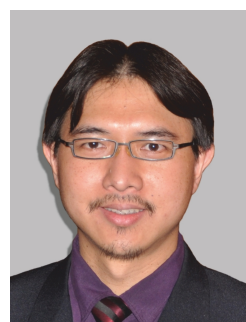

Dr Soon Xin Ng (S'99-M'03-SM'08) received the B.Eng. degree (First class) in electronic engineering and the Ph.D. degree in telecommunications from the University of Southampton, Southampton, U.K., in 1999 and 2002, respectively. From 2003 to 2006, he was a postdoctoral research fellow working on collaborative European research projects known as SCOUT, NEWCOM and PHOENIX. Since August 2006, he has been a member of academic staff in the School of Electronics and Computer Science, University of Southampton. He is involved in the OPTIMIX and CONCERTO European projects as well as the IU-ATC and UC4G projects. He is currently an Associate Professor in telecommunications at the University of Southampton.

His research interests include adaptive coded modulation, coded modulation, channel coding, space-time coding, joint source and channel coding, iterative detection, OFDM, MIMO, cooperative communications, distributed coding, quantum error correction codes and joint wireless-and-optical-fibre communications. He has published over 200 papers and co-authored two John Wiley/IEEE Press books in this field. He is a Senior Member of the IEEE, a Chartered Engineer and a Fellow of the Higher Education Academy in the UK. 